ISSN 26956462

\title{
OPINIÓN
}

\section{La importancia de la inversión de riesgo corporativo para superar el valle de la muerte en la transferencia tecnológica universitaria}

\section{Andrea Alunni'}

\section{Introducción}

Actualmente en un ecosistema clásico de innovación, las oficinas de transferencia tecnológica (TTS, por sus siglas en inglés) de la universidad estarían buscando aumentar las inversiones del sector privado (i.e. Corporaciones de los varios sectores industriales tales como el farmacéutico, digital, manufacturero, etc.) para avanzar las actividades de investigación basadas en la propiedad intelectual (PI) hacia el mercado. Del otro lado, las Corporaciones también estarían buscando oportunidades de inversión en nuevas empresas (startups) que coincidan con sus actividades de estrategia corporativa basadas en la innovación. Esto parece ofrecer la combinación perfecta para una colaboración universidad-industria exitosa. Sin embargo, al observar los impulsores que motivan esta relación, es posible distinguir que las universidades y las corporaciones tienen sea diferentes perspectivas sobre temas comunes que afectan su relación. Estas diferencias se hacen evidentes, por ejemplo, cuando a un proyecto universitario de investigación se le agota la subvención del gobierno y el investigador aún no ha alcanzado obtener financiación externa de inversores privados para convertirla en una startup. Sin esta inversión externa, el recorrido de la investigación universitaria hacia el mercado para transformarse en producto o servicio social y económicamente útil, pero que aún no es

\footnotetext{
1 Director de programa académico de innovación de Oxford e investigador en la Università di Perugia - Italia (CIRIAF) con 20 años de experiencia en la ejecución de proyectos de formación y en el campo de la gestión de patentes, la transferencia tecnológica y la financiación de la prueba de concepto para instituciones líderes como la Comisión Europea, Telecom Italia y Nomura Bank.
} 
comercialmente viable, puede detenerse. A esta falta de fondos en el desarrollo de un proyecto se le llama "valle de la muerte" y representa el tema central de la discusión de este capítulo.

\section{El problema}

Por definición, el "valle de la muerte" se refiere al período de tiempo que va desde que una startup recibe una contribución financiera inicial hasta cuando comienza a generar ingresos. Ese llamado "valle de la muerte" es, por lo tanto, una metáfora utilizada a menudo por los inversores de capital de riesgo corporativo (CVC, por sus siglas en inglés) para describir la brecha de financiación que existe entre la investigación universitaria y su comercialización (Gulbrandsen, 2009). Esto surge cuando los inversores del sector privado no captan una idea científica comercializable por su alto riesgo a sucumbir debido a que su PI todavía no se ha "aplicado" completamente y porque sus niveles de preparación tecnológica (TRL, por sus siglas en ingles) son demasiado bajos, generando así una brecha financiera que impide el progreso de la comercialización de esta idea hacia el mercado (Rasmussen y Sørheim, 2012). Tal brecha de financiación, por lo tanto, aumenta la dificultad de cubrir el flujo de caja negativo en las primeras etapas de puesta en marcha de una startup antes de que su nuevo producto o servicio genere ingresos de clientes reales.

Por lo tanto, no es sorprendente que las startups universitarias en las primeras etapas de desarrollo encuentren a menudo la brecha financiera que limita su capacidad tanto para innovar como para comercializar sus productos o servicios, y terminen en el 'valle de la muerte' (Hudson y Khazragui, 2013). Además, como para cualquier nueva empresa, a la brecha financiera se agregan también el limitado capital humano, la alta incertidumbre en términos de producto y mercado, el proceso de desarrollo volátil y los lazos de asociación débiles como los mayores impedimentos para un desarrollo exitoso de las startups (Fielden, Davidson y Makin, 2000).

\section{EI CVC como contribuyente y beneficiario del proceso de transferencia tecnológica universitaria}

A continuación, se presentan un análisis centrado en la relación que existe entre el nivel de inversión de capital de riesgo corporativo (CVC) y la capacidad que pueden tener las oficinas de transferencia tecnológica (TTO universitarias, en este 
caso) en avanzar una idea científica comercializable desde la universidad hasta el mercado.

En este escenario, el CVC se presenta como un contribuyente valioso para las nuevas empresas universitarias, tanto para llenar el vacío financiero (que queda cuando se agota la subvención para investigación que le dio el gobierno) como para proporcionar otros servicios de valor agregado tales como apoyo tecnológico, gerencial y contactos (Hellmann, Puri 2002; Dubocage, Rivaud-Danset y Redi, 2012; Caselli et al., 2009; Bertoni y Tykvova, 2012). Por lo tanto, para una nueva empresa (startup) la financiación de un CVC puede desencadenar no solo el crecimiento de la empresa como tal sino también el desarrollo de sus productos, del espíritu empresarial (Savaneviciene, Venckuviene y Girdauskiene, 2015) y de la mejora de la competitividad que llevan a cada etapa del proyecto de puesta en marcha (creación de prototipos, lanzamiento, refinanciación y resultados del proyecto), todos factores clave para su desarrollo futuro (Lantz, Sahuty Teulon, 2011).

A cambio de una tal financiación, el CVC también recoge grandes ventajas para su empresa matriz, en cuanto se beneficia de una oportunidad de inversión en una cartera diversificada, lo que permite reducir el riesgo de innovación al mismo tiempo que mantiene un cierto control desde la fase inicial de la nueva empresa con una opción de compra de la innovación una vez que la startup ha superado la etapa de emergencia. A partir del análisis empírico que Weber \& Weber (2007) llevaron a cabo entre las unidades de capital de riesgo corporativo (CVC) alemanas y sus innovadoras compañías de cartera (PC, por sus siglas en ingles) se muestra que el éxito de la PC tiene una doble importancia en términos de: altos rendimientos para la unidad CVC y potencial estratégico para la innovación radical para la casa matriz. Esto corrobora que el financiamiento de nuevas empresas universitarias por parte de un CVC ofrece un modelo para que las grandes corporaciones continúen desarrollándose en el sector de alta tecnología.

\section{El impacto del CVC como catalizador del valle de la muerte}

El impacto que tiene la inversión del CVC en el desarrollo de las funciones de una TTO universitaria se encuentra caracterizado por el mecanismo de intercambio de recursos. Lo adoptan las principales universidades de los países desarrollados (como Oxford, Cambridge, Harvard, MIT, etc.) como medio para otorgar a sus nuevas empresas universitarias (startups) acceso a recursos, por lo general, en la fase inicial del proceso que lleva las ideas científicas universitarias a modelos co- 
merciales reales, al mismo tiempo que ofrecen a las corporaciones la oportunidad de acercarse al ecosistema emprendedor universitario. En el caso de universidades prominentes, este mecanismo resulta suficiente debido fundamentalmente al hecho de que el presupuesto universitario de investigación respalda ampliamente la actividad de investigación realizada. En el caso de la Universidad de Oxford, por ejemplo, la investigación en todas las materias está financiada principalmente por la propia universidad (con un presupuesto anual de investigación universitaria cercano a $£ 600$ millones (Isis Innovation Report, 2015). En el caso de universidades que no cuenten con un presupuesto de estas dimensiones, mecanismos adicionales (hackathons, venture builders, corporate accelerators, etc.) deberían también ser contemplados e inclusive reforzados a través de una colaboración separada entre las escuelas de negocios universitarias y la industria.

En el intento de medir el impacto que tiene la inversión de capital de riesgo corporativo en el campo de la transferencia tecnológica universitaria, continuaremos a referir al mecanismo de intercambio de recursos tomando el caso de la TTO de la Universidad de Oxford como un buen ejemplo para presentar cómo funcionan las dos modalidades de inversión de riesgo en el campo de la transferencia de tecnología que allí se adoptan sea de manera directa o indirecta por parte del CVC. Es decir: (a) cuando la colaboración se desarrolla de forma directa-externa, la CVC sin usar un nuevo fondo de riesgo dedicado adquiere (o toma una posición de capital) una nueva empresa universitaria (startup) adoptando una posición minoritaria junto con la universidad y el equipo directivo, para hacer crecer un negocio a su externo que pueda beneficiar directamente los resultados financieros corporativos; y (b) cuando la colaboración se desarrolla de forma indirecta-externa, la corporación invierte en un fondo de capital de riesgo que se dirige a empresas externas (en este caso startups universitarias) en industrias específicas o sectores tecnológicos, obteniendo así indirectamente acceso y participación a nuevos negocios que pueden ser absorbidos en la casa matriz en el futuro a través de operaciones de fusiones y adquisiciones.

Del lado de la Universidad, el mejor impacto lo obtiene cuando la colaboración con el CVC ha facilitado ulteriormente el esfuerzo hecho por el TTO para obtener una cartera de IP debidamente protegida y desarrollada en nuevas empresas para luego ser comercializadas. Lo que significa que la colaboración CVC-TTO, en el caso de Oxford, manifiesta la cultura empresarial de la universidad y su entorno (a nivel nacional, regional y local) para establecer los necesarios mecanismos de colaboración con las Corporaciones que ocurren cuando la TTO hace presentación temprana de su IP a las empresas que deseen desarrollar tanto la tecnología como el modelo comercial de la nueva invención. Se puede decir por tanto que el 
impacto es esencial en el esfuerzo de hacer cruzar el valle de la muerte por parte de las startups universitarias, para lo cual el TTO de la Universidad de Oxford ayuda ulteriormente con el desarrollo de planes de negocios en colaboración con su escuela de negocios (Said); ofrece espacio para la incubación de estas empresas tecnológicas universitarias (The Foundry) donde existe un espacio de trabajo conjunto permanente que también permite a la universidad explorar otros mecanismos de $\mathrm{CV}$, incluidos hackatones, cajas de burbujas (equipos de exploración), excubadoras y premios de desafío. Por último, pero no menos importante, el TTO también se afianza al ecosistema emprendedor de Oxford para proporcionar a las nuevas empresas universitarias interconexiones estrechas con los parques científicos, los fondos semilla y de riesgo.

\section{Cuestiones que afectan la colaboracion CVC-TTO}

A la luz de lo anterior, surge una pregunta ordinaria: si el financiamiento de CVC es un catalizador propicio para que las nuevas empresas (startups universitarias) superen el "valle de la muerte" y las startups son una gran fuente de innovación para las grandes corporaciones (o casas madres): ¿por qué la falta de financiación inicial por parte del CVC todavía está causando una alta mortalidad a un gran número de startups universitarias?

Para encontrar una respuesta, es posible que debamos analizar las cuestiones que afectan esta relación. Al observar los impulsores que motivan la relación universidad-industria, es posible distinguir que las TTOs y los CVCs tienen diferentes perspectivas sobre temas comunes.

Basándose en investigaciones que analizan la colaboración entre la universidad y la industria, algunos han señalado repetidamente el problema que surge del diferente horizonte temporal en el que la universidad y las empresas operan, invierten y planifican sus actividades; revelando que el enfoque de la investigación académica en desafíos a largo plazo a menudo entra en conflicto con el enfoque a corto plazo en los objetivos financieros de los programas de CVC. Como resultado, los CVCs a veces encuentran que las universidades son demasiado lentas y demasiado burocráticas para ser buenos socios (Perkmann \& Salter, 2012) y muestran una reticencia persistente a invertir en proyectos universitarios, considerando además el agravante de que tener una participación accionaria en startups (en general) es una inversión de alto riesgo que a menudo conduce a un activo ilíquido a largo plazo (Wright, Lockett, Clarysse \& Binks, 2006). Esto último, sin embargo, 
ha sido descartado por autores como Ernst, Witt y Brachtendorf (2005) quienes en analizar los beneficios estratégicos a largo plazo de los CVC al colaborar con universidades corroboraron que el riesgo de que tales beneficios no resulten evidentes existe porque el horizonte temporal de evaluación no lo permite. Es decir, si las actividades de inversión de un CVC en una startup universitaria tienen un efecto positivo a largo plazo (i.e. proporciona altos rendimientos para la unidad CVC y potencial estratégico para la innovación radical para la casa matriz), estas ganancias estratégicas a menudo no son evidentes porque normalmente el rendimiento de un CVC se evalúa en función del corto período.

Otras también observan que las TTO y los CVC también presentan diferentes perspectivas en tema de expectativas cuando diseñan la colaboración en proyectos comunes. Aquí, de nuevo, se pone de manifiesto una reticencia por parte de los CVC a invertir en los proyectos universitarios, en particular cuando estos han iniciado con fondos públicos y desean desarrollar aún más los hallazgos con fondos privados (de CVC, en este caso), según Wright, Lockett, Clarysse \& Binks (2006) y Ernst, Witt y Brachtendorf (2005) como consecuencia de un prejuicio infundado. En particular, porque la investigación impulsada por la universidad, aunque más riesgosa, permite logros científicos y tecnológicos inesperados y fructíferos, con grandes repercusiones en otros campos. Mientras que los proyectos impulsados por la industria, en cambio, resultan en logros más modestos, pero es más probable que sean adoptados para su uso por empresas que invierten en la transferencia de conocimiento a través de otros canales que no son universitarios (Bekkers y Bodas-Freitas, 2010).

Esto nos permite enumerar las principales expectativas de colaboración entre la universidad y la industria y determinar en qué difieren. Desde el punto de vista del CVC, las principales aspiraciones a invertir tienen un enfoque más fuerte en los objetivos estratégicos corporativos determinados por su creciente necesidad de crecimiento adicional en los negocios impulsados por la innovación (BCG, 2019). Mientras que, desde la perspectiva de una $\Pi O$, el primer paso para el éxito implica, por lo general, formar una sociedad con una corporación (CVC en este caso) y una nueva empresa (spin-off universitaria), donde la $\Pi O$ construye el puente que conduzca la investigación al mercado y genere dinero para la institución, a veces con un académico en el centro. Eso, naturalmente, puede involucrar otros servicios que ofrece el $\Pi \mathrm{TO}$ tales como asistencia para llevar a cabo una investigación de mercado, proporcionar espacio para incubadoras y/o financiar el experimento de prueba de concepto o registrar patentes. 


\section{Los mecanismos de colaboración entre CVC-TTO}

El grafico a continuación representa los cuatro más importantes mecanismos de intercambio de recursos que están emergiendo en una colaboración industria-universidad (CVC-TOO), en función del horizonte de tiempo y la cantidad de dinero invertido por CVC. El mismo, muestra claramente que la inversión clásica de CVC tiende a favorecer colaboraciones que evolucionan en el corto plazo, en contraste con el enfoque universitario cuyo objetivo de colaboración es el de reforzar su relación con el CVC para actividades a largo plazo (incluidos POC y empresas de asociación estratégica) para una colaboración exitosa.

\section{FIGURA I}

\section{Emerging collaboration between CVCs and TTOs Taking into account time-horizon and money invested}

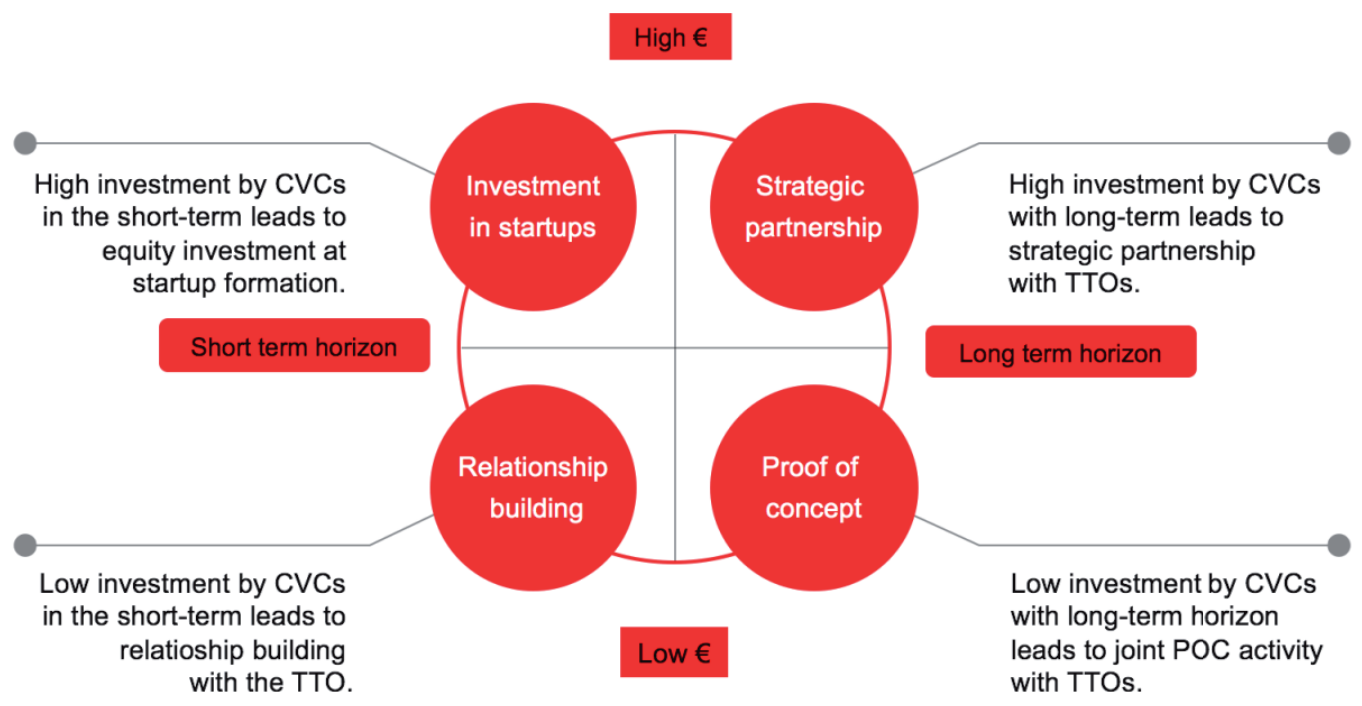

\section{Es necesario un enfoque transversal}

Sería fácil pensar que las colaboraciones CVC-TTO se mantienen o abandonan simplemente en función del rendimiento, pero no es tan simple. Las unidades corporativas de capital de riesgo a menudo se ejecutan por sus beneficios estratégicos, que son mucho más difíciles de evaluar que los retornos financieros puros 
(Gaba, 2017). Esta incertidumbre hace que el enfoque también en los beneficios intangibles sea importante.

Los problemas identificados revelan que es importante adoptar un enfoque transversal para abordarlos en programas conjuntos donde los CVC y las TTO pueden comunicar sus necesidades y alinear sus perspectivas con el fin de garantizar que estos dos mundos e individuos de estos dos lados puedan cooperar mejor.

Para generar una lista común de objetivos de CVC-TTO y luego priorizar los que son más importantes para cada circunstancia, es importante comprender que la cooperación fructífera implica un cambio de mentalidad y que, en consecuencia, esto tendrá un impacto en el horizonte de tiempo corporativo y los objetivos estratégicos que usan para operar e invertir juntos.

Para alentar la colaboración entre la universidad y la industria se pueden avanzar algunas soluciones para abordar los problemas existentes, y hacer que sus relaciones se hagan más valiosas, basadas en enfoques que han sido adoptados con éxito por universidades destacadas para promover una relación efectiva. Esto comprende dos acciones estratégicas complementarias que, por una parte, se centra sobre las personas para construir relaciones para alinear las diferentes perspectivas institucionales, $y$, por otra, en los procesos para forjar mecanismos de colaboración conjunto que permita eficazmente ayudar a las startups a cruzar el valle de la muerte.

\section{I. Construir relaciones para alinear perspectivas}

La colaboración entre la industria y la universidad no siempre es efectiva; para que funcionen, cada parte deben superar la brecha cultural y de comunicación que comportan un daño a las colaboraciones industriales y universitarias de todo tipo y que socavan su potencial.

\section{I.I. Capacitación para alinear el horizonte temporal y los objetivos estratégicos}

Una cooperación efectiva requiere un cambio de mentalidad para alinear el horizonte temporal y los objetivos estratégicos que las TTO y CVC utilizan para operar e invertir juntos. Este cambio puede ser ayudado con capacitación común para crear un amortiguador contra la brecha que los separa en términos de lenguaje, perspectiva y expectativas. Esencialmente, a través de la capacitación de 
los gerentes de transferencia de tecnología de las $T$ O universitarias obtendrán las habilidades necesarias para comprender y negociar los DPI con las corporaciones, mientras que las corporaciones obtendrán una comprensión perspicaz sobre la novedad y las posibilidades de que las derivaciones universitarias impliquen sus objetivos financieros como beneficio de la innovación externa.

La revisión de la literatura sugiere que la universidad y la industria perciben de manera diferente sus barreras habituales para la colaboración (Bierregaard, 2010), ya que las diferencias culturales entre empresas y universidades tienden a impedir el intercambio de conocimientos e imponen desafíos al control del proyecto si no se abordan adecuadamente. A través de la capacitación, las posibilidades de experimentar una convergencia institucional pueden constituir un espacio cultural compartido para el intercambio de conocimiento y la comunicación en los proyectos conjuntos de CVC-TTO y poder utilizar las habilidades sociales adquiridas para cerrar las brechas institucionales percibidas.

Esto lo confirman Bruneel, d'Este y Salter (2010), quienes descubrieron que los obstáculos a las colaboraciones entre las universidades y la industria se ven afectados por la experiencia de colaboración, la amplitud de la interacción y la confianza entre organizaciones para reducir los diferentes tipos de barreras. En particular, la experiencia previa de investigación colaborativa reduce las barreras relacionadas con la orientación y que mayores niveles de confianza reducen ambos tipos de barreras estudiadas. También indican que la amplitud de la interacción disminuye la orientación relacionada con la orientación, pero aumenta las barreras relacionadas con la transacción. La confianza entre organizaciones es, por lo tanto, uno de los mecanismos más fuertes para reducir las barreras a la interacción entre las universidades y la industria. Por esta razón, el sistema tradicional de reciprocidad e intercambio informal, que dominó la interacción CVC-TTO, debería ser un aspecto importante a tener en cuenta en el intento de apoyar y construir colaboraciones duraderas.

Al observar la naturaleza y el impacto de las universidades y otras instituciones de educación superior (IES) en la innovación y el crecimiento de las empresas, Howells, Ramlogan y Cheng (2012) encontraron que la colaboración varía significativamente entre el tipo de empresa involucrada y su ubicación, y que la naturaleza y los efectos de dicha colaboración son, como cabría esperar, contradictorios. En realidad, los investigadores industriales que tienen poca experiencia en interactuar con la universidad. Ellos son más propensos a reportar grandes barreras para la colaboración (es decir, diferentes marcos y dificultades para identificar, ubicar y acceder al conocimiento universitario). Al contrario, aquellos investigadores industriales, 
que tienen más experiencia en colaborar y establecer contactos con investigadores universitarios y en escanear y buscar publicaciones académicas para informar sus actividades de I + D industrial, ven menos barreras. Además, los investigadores industriales que han estado involucrados intensamente en patentar e interactuar con universidades a través de TTO a menudo enfatizan las preocupaciones sobre los problemas de propiedad de los DPI o los altos costos de gestión.

La naturaleza de la capacitación que requiere la colaboración de CVC-TTO.

- Estas capacitaciones deben diseñarse para tres audiencias diferentes: (i) una solo para TTO, (ii) otra para Corporate Ventures y (iii) una articulación para TTOs y Corporate Ventures.

- El plan de estudios debe cubrir los siguientes temas: DPI, negociaciones de acuerdos de licencia y planes de desarrollo de PI.

- Estas capacitaciones deben ser pensadas por instituciones gubernamentales relevantes, universidades y asociaciones de profesionales (como AUTM en EE. UU., ASTP-PROTON en la UE y PRAXI-Único en el Reino Unido).

- La capacitación debe comenzar con un formato amigable para la enseñanza (aprendizaje en el aula, tutoriales de e-learning y capacitación local y / o regional) que se puede reforzar con pasantías y tutorías para ayudar a construir relaciones de trabajo sostenidas, así como perspectivas de carrera.

\section{I.2. Capacitación para que la colaboración sea más eficaz y duradera}

Para que la colaboración CVC-TTO sea eficaz, es necesario que la relación sea duradera entre estos dos tipos de organizaciones y eso implica hacer un esfuerzo para alinear el uso conjunto de talentos y recursos.

Si bien las universidades que operan en un ámbito de innovación cada vez más diversificado y rápidamente cambiante, para hacer más atractivas sus actividades de transferencia tecnológica claramente requerirán un gran esfuerzo de desarrollo de capacidades para equipar a los actores de transferencia de tecnología con las habilidades y recursos necesarios para garantizar el éxito. Por su parte la industria, necesitará siempre más invertir para que los gerentes de una unidad de CVC acumulen experiencia y confianza en tema de transferencia tecnológica, reduciendo su propensión natural a seguir ciegamente a la multitud cuando se trata de decidir dónde invertir. 
En tema de recursos, es necesario también reconocer que una colaboración eficaz sirve tanto a las universidades como a las empresas. Es decir, para la industria es indiscutible que son las universidades el lugar donde se puede obtener la oportunidad de ver en primera línea la próxima gran idea. De hecho, las empresas que están alineadas con la investigación en etapas tempranas ven signos tempranos de la próxima gran oportunidad, y recibirán una ventaja sobre la competencia. Las empresas también se benefician del acceso a una red de profesores, líderes de opinión clave y científicos líderes, y la capacidad de asociación con otras compañías involucradas en investigación sobre temas de interés común. Finalmente, colaborar con las universidades permite a las corporaciones "reducir el riesgo" de su investigación y soslayar costos de investigación, ahorrando dinero incluso mientras canalizan recursos financieros a las universidades.

Para las universidades, es fundamental comprender cómo desarrollar y maximizar las colaboraciones corporativas en este entorno de financiación incierto. Las colaboraciones industriales pueden decir a los académicos dónde se encuentran los desafíos actuales y las asociaciones mutuamente beneficiosas pueden producir investigación e innovación innovadoras que resuelven problemas complejos, impulsan el crecimiento económico y crean una fuerza laboral más calificada. También ayudamos a los investigadores / inventores universitarios a construir su investigación basada en una experiencia del mundo real. Además, mientras que las universidades y la industria disfrutan de una relación simbiótica, la sociedad también se beneficia del crecimiento económico a la sociedad impulsado por la innovación y la fuerza laboral capacitada.

El desafío es encontrar formas en que las $T T O$ y los CVC puedan comunicar sus necesidades para encontrar un terreno común. A continuación, se ha identificado un par de opciones estratégicas para prolongar una mejor colaboración:

\section{I.3. Elección apropiada del personal}

Según Gaba (2017), las elecciones específicas de personal y los altos niveles de inversión pueden prolongar la vida útil de las unidades corporativas de capital de riesgo.

Dado que CVC se ha convertido en una herramienta crucial para las grandes corporaciones bajo presión para innovar más rápido, la colaboración efectiva con las $\mathrm{OT}$ les brinda una ventana a nuevas tecnologías y una forma de acceder a ideas novedosas, especialmente del tipo que podría arruinar las obras. Sin em- 
bargo, para que esto suceda, los CVC deben mantener el rumbo para cosechar los beneficios. Esto no siempre es notorio para las empresas y, a pesar de los beneficios obvios, las unidades CVC tienden a tener una vida útil corta. Es decir, entre 1980 y 2006 , el ciclo de vida de un programa de empresas corporativas se ha estimado en aproximadamente cuatro años.

Parece que el aprendizaje y el abandono de las prácticas corporativas de capital de riesgo son factores importantes que llevaron a las corporaciones a abandonar sus unidades CVC y que las corporaciones que desean mantener su unidad a largo plazo deberían pensar cuidadosamente sobre las opciones de implementación que toman, en particular la dotación de personal, ya que pueden estar sembrando inadvertidamente las semillas del abandono (Gaba y Dokko, 2016). Por ejemplo, después de controlar múltiples variables, incluido el colapso de las puntocom de 2000, estos escritores descubrieron que contratar al menos a un miembro del equipo con experiencia previa en capital de riesgo hace que las empresas tengan menos probabilidades de abandonar su unidad CVC. Aquí el abandono se define como la ausencia de inversiones de capital riesgo durante al menos cuatro años.

Del lado de las TTO universitarias que operan en este panorama de innovación cada vez más diversificado y rápidamente cambiante, también se requerirá claramente un gran esfuerzo de creación de capacidad para equipar a sus gerentes de transferencia de tecnología con las habilidades y recursos necesarios para interactuar con las empresas y superar malentendidos y percepciones erróneas en común cuestiones. Se había teorizado que los $\Pi$ calificados aportan un conocimiento profundo de la práctica y que una $\Pi \mathrm{TO}$ tendría una mayor probabilidad de éxito si contara con personas íntimamente familiarizadas con la capacidad inherente de integrar tecnologías de inicio y gestionar las expectativas de la alta dirección. Resulta que contar con un equipo calificado de TTM con experiencia en capital de riesgo a bordo aporta un conocimiento profundo de la práctica, como un mejor manejo de cómo buscar nuevas ideas, cómo evaluar nuevas empresas en etapa temprana y crear contratos, etc. Sin El conocimiento y la mentalidad completos, los equipos que carecen de experiencia con capital de riesgo tienden a adaptar las prácticas de capital riesgo de manera inútil.

\section{I.4. Evaluación efectiva de las compensaciones}

Como se mencionó anteriormente, no tiene pretensiones pensar que las prácticas se mantienen o abandonan simplemente en función del rendimiento. Las unidades de capital de riesgo corporativas deben prestar atención al evaluar sus beneficios 
estratégicos, que son mucho más difíciles de evaluar que los retornos financieros puros. Además de garantizar que las inversiones de capital riesgo se realicen correctamente, es más probable que los miembros del equipo con experiencia en capital de riesgo enfaticen los rendimientos financieros. Si la empresa se preocupa por los retornos estratégicos, la contratación de personas con experiencia real en capitalismo de riesgo es esencial para acumular experiencia y confianza en sí mismo, reduciendo su propensión natural a seguir ciegamente a la multitud.

Los programas corporativos de capital de riesgo pueden desempeñar un papel estratégico para mantenerse al día con las interrupciones en el negocio y garantizar su longevidad. Específicamente, se establece que las unidades CVC que hicieron más que el número medio de rondas de inversión en las empresas tenían menos probabilidades de ser abandonadas, incluso después de tener en cuenta la presión social negativa. Teorizamos que más inversiones permiten a los gerentes perfeccionar su experiencia y aumentar su confianza en sí mismos, disminuyendo su instinto de seguir a la multitud.

\subsection{Afianzar mecanismos de colaboracion para cruzar el valle de la muerte}

En el panorama general de la transferencia de tecnología, cuando las personas que conforman los grupos de TTO universitarios y de empresas corporativas interactúan, con mucha frecuencia las corporaciones buscan la colaboración casual. Según Perkmann y Salter (2012) este tipo de carencia actúa como un freno a la oportunidad de entenderse para lograr mejores resultados trabajando juntos y elegir mecanismos apropiados de colaboración.

En la elección de un mecanismo apropiado de colaboración, por ejemplo, las empresas deberían responder preguntas como ¿Qué pueden hacer estas TTO universitarias mejor o diferente de lo que podemos hacer nosotros? De hecho, de manera utilitaria, los principales CVC tienden a invertir en áreas de investigación universitaria donde todavía no tiene sentido comercial para la casa matriz desarrollar una amplia capacidad tecnológica. En este caso, un Director de TTO podría considerar la adopción de un mecanismo que le permita movilizar recursos para ayudar a las empresas a abordar la pregunta. Tal mecanismo permitiría iniciar un ciclo virtuoso donde tanto el grupo TTO de la universidad como el del CVC puedan reunirse periódicamente, por ejemplo, para discutir sobre las nuevas IP universitarias desarrolladas en campos específicos que son estratégicos para la empresa matriz, pero sobre las cuales el CVC puede tener falta de conocimiento, por lo que pudiera desplegar un interés en alearse con la TO como socio estratégico 
para tener la oportunidad de iniciar nuevos negocios basados en IP universitarias cuando surja la oportunidad adecuada para la casa matriz.

\subsection{Alianza estratégica e inversión en startups universitarias}

Una alianza estratégica puede ser considerada por una empresa como un mecanismo para facilitar la provisión de fondos de innovación a una TTO a través de un programa conjunto universidad-industria para desarrollar pruebas de concepto (POC). Dicho programa puede estructurarse en torno a la fase de desarrollo de la investigación universitaria hasta la entrega de una prueba de concepto que funcione para validar mediante pruebas piloto el potencial de comercialización que idealmente se convierte en un negocio rentable. Para lograr una evaluación efectiva de esas oportunidades, un equipo conjunto debe establecer criterios de evaluación apropiados, definir "áreas de interés" comunes e incluir personal con experiencia técnica y de mercado relevante en el comité de evaluación. Visto que la fase de comercialización a menudo conlleva riesgos de inversión significativos sería pertinente, por lo tanto, poner el énfasis en la validación a través de la prueba de concepto para reducir las incertidumbres técnicas y de mercado al obtener comentarios tempranos de los clientes $y$, a su vez, emitir un juicio bien informado sobre la probabilidad de éxito comercial (Burgers, et al., 2010).

Según Elmuti, Abebe y Nicolosi (2005), las principales ventajas para la comunidad académica (financiación de la investigación y oportunidades prácticas de aprendizaje para estudiantes) y para la industria en la creación de alianzas estratégicas, es también aquella de reducir los costos de investigación y desarrollo y aumentar las oportunidades de transferencia de tecnología que aumentan la competitividad. Los inconvenientes, como mencionado antes, pueden incluir las diferencias culturales y valores de trabajo de los socios. Por lo tanto, las alianzas universidad-industria deben estar respaldadas por procesos continuos de aprendizaje y reestructuración para superar tales diferencias.

\subsubsection{POC como valioso y principal mecanismo para aumentar el valor de} las startups universitarias

Según Vass (2008), el trabajo de Audretschs sanciona cómo el aumento sin precedentes del gasto en investigación y desarrollo (I + D) en los Estados Unidos durante y después de la Segunda Guerra Mundial se convirtió en actividad económica al operar muchos centros de prueba de concepto de transferencia de tecnología. Con 
base en esta experiencia excepcional, señala, un centro de prueba de concepto efectivo debe ser capaz de encontrar: (i) una combinación de equipo administrativo y asesores que sean ejes en la red local de la industria tecnológica; (ii) un personal conocedor de $\Pi \mathrm{T}$, que en realidad puede ser más útil para acelerar la comercialización de tecnología universitaria que la financiación inicial; y (iii) una red social fuerte, si existe en la comunidad circundante, que incluye asesores, inversores ángeles, capitalistas de riesgo y empresas interesadas para que los beneficiarios se asocien.

Es por tanto necesario destacar que la etapa de $\mathrm{POC}$ es uno de los hitos más valiosos para una startup. Ya sea que el proceso de POC implique el desarrollo de un prototipo, la construcción de un algoritmo o la evaluación de la demanda del mercado, la etapa de POC demuestra a los fundadores de inicio y a los posibles inversores si existe o no un mercado para su producto en primer lugar.

La etapa de POC no es solo una cuestión de tomar una idea y darle vida, sino que muestra potencial, particularmente a los inversores que consideran respaldar un proyecto, al mostrar que los posibles usuarios pueden realmente interesarse, usar y disfrutar el producto. Ejecutar un POC exitoso da validez a que tal necesidad existe y que el producto puede proporcionar la solución. Confirmar el valor de una startup para el mercado brinda a los diferentes equipos de la compañía el impulso que necesitan para expandir la influencia de su tecnología innovadora.

Las nuevas empresas tienen muchas ventajas de la creación de prototipos, incluida la capacidad de adaptarse al cambio, pensar creativamente, ideas de arranque. Esto es crucial cuando hay una breve línea de tiempo para demostrar el éxito, muchas veces como resultado de este problema exacto. Las startups universitarias pueden usar el impulso de un POC exitoso para seguir avanzando sus ideas científicas hacia el mercado. Además, para las nuevas empresas en la etapa inicial, mostrar los resultados de una prueba de concepto exitosa puede aumentar su valoración y ayudar a sus esfuerzos para asegurar futuras rondas de inversión. Para las nuevas empresas que actualmente están a punto de lanzar una ronda de inversión, una POC exitosa no solo puede aumentar su valor, sino que también puede convencer a los inversores independientes de que tiene un claro ajuste en el mercado de productos.

Dado que el proceso de POC es complejo, cuando se trabaja en una demostración es necesario que el proceso sea confiable y amigable con la innovación. Aquí es cuando un soporte conjunto CVC-TTO se vuelve instrumental, ya que puede conciliar la incapacidad de la startup para llegar a la persona adecuada, ya sea 
por falta de conexiones, fondos o tiempo de su parte, que a menudo bloquea las startups y dificulta su capacidad de integrar su solución en la empresa Además, la conexión oportuna de inicio a las empresas en un punto tan crítico en su etapa de I + D puede llevar los beneficios de dicha colaboración a todas las rondas de financiación futuras.

POC también aborda el problema común que surge cuando el sector privado / los inversores no captarán una idea científica comercializable porque es demasiado arriesgado (ya que todavía no se ha "aplicado" completamente y sus niveles de preparación tecnológica (TRL) son demasiado bajos) generando una brecha financiera que impide el progreso de esta comercialización de la idea (Rasmussen y Sørheim, 2012). POC aquí ofrece una actividad conjunta efectiva que pueden ser promovidas por TOs y CVC juntas para ayudar a las nuevas empresas a cruzar el "valle de la muerte".

\section{Recomendaciones}

Para abordar los desafíos que presenta actualmente la colaboración entre la universidad y la industria, es necesario saber que el impacto positivo de esta colaboración dependerá en gran medida de la capacidad del CVC de abrazar activamente a las actividades de transferencia tecnológica impulsadas por las TTO universitarias utilizando las diferencias entre la industria y la academia para crear alianzas estratégicas que permitan en el tiempo alinear objetivos comunes.

Las empresas por su parte pudieran considerar hacer un esfuerzo en ajustar gradualmente sus expectativas de beneficios a partir de la innovación externa que esperan recibir al colaborar con las universidades para que su enfoque (a corto plazo centrado) sobre objetivos financieros pueda alcanzar el tiempo de producir los beneficios de medio-largo periodo donde se pueda medir el crecimiento empresarial impulsado por la innovación.

Asegurarse de que el impacto de CVC en el papel evolutivo de las TTO universitarias en el campo de la transferencia de tecnología puede amplificarse aún más mediante el establecimiento de un mecanismo de Prueba de Concepto (POC) que asegure una cartera de proyectos (basados en IP) confiable para los inversores en etapa temprana para un ecosistema creciente impulsado por la innovación. 
Reconocer en el POC un poderoso instrumento que proporciona fondos transparentes, seguros y no competitivos para desarrollar prototipos, demostraciones prácticas y experimentos cruciales necesarios para traducir patentes o elementos de propiedad intelectual en productos comercializables.

Forjar una cooperación efectiva entre TTO y CVC basada sobre una relación duradera entre estos dos tipos de organizaciones y donde exista la capacidad de encontrar un terreno común para que las OTT y los CVC puedan comunicar sus necesidades y cada parte supere la división cultural y de comunicaciones que tiende a perjudicar y socavar el potencial de un trabajo conjunto, permite impulsar la capacitación conjunta de universidades y corporaciones para abordar problemas comunes y mejorar las posibilidades de encontrar un lenguaje mutuo que conduzca a mejorar resultados al trabajar juntos sobre expectativas comunes.

\section{Referencias}

BeKKERS, R., \& BodAs-FreitAs, I. (2010, June), "Catalysts and barriers: Factors that affect the performance of university-industry collaborations". In Conference paper International Schumpeter Society Conference.

BJeRreGaARD, T. (2010), "Industry and academia in convergence: Micro-institutional dimensions of R\&D collaboration". Technovation, 30(2), 100-108.

Bruneel, J., D'Este, P., \& Salter, A. (2010), "Investigating the factors that diminish the barriers to university-industry collaboration". Research policy, 39(7), 858-868.

Burgers, H., Shah, C., \& SCHOlten, V. (2010), "Managing a portfolio of corporate venture units: challenges in achieving within-unit focus and between-unit coordination". Proceedings of the 7th AGSE International Entrepreneurship Research Exchange, 361-370.

Ernst, H., WitT, P., \& Brachtendorf, G. (2005), "Corporate venture capital as a strategy for external innovation: an exploratory empirical study". r\&d Management, 35(3), 233-242.

GABA, V. (2017, April 26), "Why Do So Many Corporate VCs Die Young?" [Online article:Entrepreneurship].ISEAD. Retrievedfromhttps://knowledge.insead.edu/entrepreneurship/why-do-so-many-corporate-vcs-die-young-5951\#Xh+OKvljxJ6vz 1 bf.99 
GABA, V., \& DoKKo, G. (2016), "Learning to let go: Social influence, learning, and the abandonment of corporate venture capital practices". Strategic management journal, 37(8), 1558-1577.

GULBRANDSEN, K. E. (2009), Bridging the valley of death: The rhetoric of technology transfer.

Howells, J., Ramlogan, R., \& Cheng, S. L. (2012), "Innovation and university collaboration: paradox and complexity within the knowledge economy". Cambridge Journal of Economics, 36(3), 703-721.

Lantz, J. S., Sahut, J. M., \& Teulon, F. (2011), "What is the real role of corporate venture capital?". International Journal of Business, 16(4), 367.

Perkmann, M., \& Salter, A. (2012), "How to create productive partnerships with universities". MIT Sloan Management Review, 53(4), 79-88.

RASMUSSEN, E., \& SøRHEIM, R. (2012), "How governments seek to bridge the financing gap for university spin-offs: Proof-of-concept, pre-seed, and seed funding". Technology Analysis \& Strategic Management, 24(7), 663-678.

Savaneviciene, A., Venckuviene, V., \& Girdauskiene, L. (2015), "Venture Capital a Catalyst for Start-Ups to Overcome the "Valley of Death": Lithuanian Case". Procedia Economics and Finance, 26, 1052-1059.

VAsS, L. T. (2008), Unchallenged Assumptions about Innovation Meet Shibboleths of University Tech Transfer.

WeBER, B., \& WeBER, C. (2007), "Corporate venture capital as a means of radical innovation: Relational fit, social capital, and knowledge transfer". Journal of Engineering and technology management, 24(1-2), 11-35.

Wright, M., Locket, A., Clarysse, B., \& Binks, M. (2006), "University spin-out companies and venture capital". Research policy, 35(4), 481-501. 